Objectives A feasibility study has shown that a scientifically rigorous and comprehensive epidemiological study of workers involved in the manufacture or production of tungsten carbide with a cobalt binder is feasible, and should include workers from sites in the United States and in Europe. Objectives include: (i) to investigate the total and cause-specific mortality experience of current and former workers as compared with the corresponding national and local populations from which the workforces were drawn, with adjustment for potential confounding factors; (ii) to characterise as completely as possible the current and past working environment of the study participants; and (iii) to determine the relationship between level and duration of exposures and mortality from lung cancer with analytic adjustment for important potential co-exposures, including tobacco smoke. The study is funded by the International Tungsten Industry Association.

Methods The study cohort will be enumerated using human resources data, with cross-checks for completeness against pension, payroll, occupational health and other sources. All available occupational hygiene data will be extracted to facilitate a studywide exposure assessment.

Results Initial meetings have been held with the two UK factories to establish the scope and quality of demographic and industrial hygiene data. In parallel with this an application has been submitted to an ethics committee and the National Information Governance Board, the latter seeking to obtain an exemption from having to gain positive consent for study participants. A worker leaflet explaining the study has been drafted for inclusion in the ethical submission. Work to determine the optimum way to extract the data from the factories, is currently underway.

Conclusions This large study will represent multiple companies, countries and manufacturing processes and will be larger, more robust and more definitive than any hard-metal manufacturing epidemiological study done to date.

\section{INCIDENCE OF MESOTHELIOMAS IN QUÉBEC AND CANADA FROM 1984 THROUGH 2007: TRENDS AND ESTIMATE OF FUTURE BURDEN}

${ }^{1}$ Krupoves, ${ }^{2}$ Camus. 'INSPQ, Montreal, Canada; '2Université de Montréal, Montréal, Canada

\subsection{6/oemed-2013-101717.56}

Objectives We compared the incidence rates of pleural (MPL) and peritoneal (MPR) mesotheliomas between Quebec and the rest of Canada to describe past and estimate future temporal trends of MPL in Quebec until the year 2032.

Methods New cases occurring between 1984 and 2007 were counted in the Canadian Cancer Registry according to the international classification of diseases for Oncology, 3rd version (ICD-O-3). Equivalent ICD-O-1 coding was used to identify mesothelioma cases before 1992. Age-standardised rates were compared between sexes and regions. Poisson regressions were carried out to assess the effects of birth cohorts and to estimate future rates of MPL.

Results The age-standardised incidence rates of MPL averaged 2.12 and 0.42 for 100000 person-years among Quebec men and women respectively. Age-adjusted rates of MPL were 1.45 (95\% CI $=1.37-1.54)$ times higher in Quebec men and 2.00 (95\% CI $=1.76-2.27)$ times in women than among Canadian men and women. The age-adjusted rate of MPR was 1.36 (95\% CI $=1.09-1.68)$ times higher among Quebec residents than in the rest of Canada. A significant slowdown in the increase of MPL was observed after 1995. Younger cohorts experienced a lower incidence of MPL. The incidence of MPL should peak between 2008 and 2012 (at 2.79/100000 in Québec and 1.79/100000 in Canada). In Quebec, there would be an absolute excess of some 700 new male cases and 160 female cases over a 5 -year period.

Conclusions The observed higher and continuously increasing rates of MPL in Québec warrant a stricter surveillance of mesothelioma incidence and asbestos exposure to ensure that rates effectively drop down to background levels.

\section{AN INTERNATIONAL HISTORICAL COHORT STUDY OF WORKERS IN THE HARD-METAL INDUSTRY}

${ }^{1} \mathrm{M}$ Marsh, ${ }^{1}$ Buchanich, ${ }^{1}$ Downing, ${ }^{1}$ Kennedy, ${ }^{2}$ Esmen, ${ }^{3}$ Moshammer, ${ }^{4}$ Morfeld, ${ }^{5}$ Erren, ${ }^{6}$ Svartengren, ${ }^{7}$ Westberg, ${ }^{8} \mathrm{McElvenny},{ }^{8}$ Cherrie. ${ }^{1}$ University of Pittsburgh, Pittsburgh, United States of America; ${ }^{2}$ University of llinois at Chicago, Chicago, United States of America; ${ }^{3}$ Medical University of Vienna, Vienna, Austria; ${ }^{4}$ Evonick Industries, Inc., Cologne, Germany; ${ }^{5}$ University of Cologne, Cologne, Germany; ${ }^{6}$ Karolinska Institute,

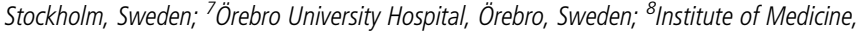
Edinburgh, United Kingdom

\subsection{6/oemed-2013-101717.57}

Objectives In 2006, IARC found limited evidence in humans and sufficient evidence in animals that tungsten carbide (WC) with cobalt binder (WCCo) acted as a lung carcinogen (Group 2A). Our historical cohort study was designed to overcome certain limitations of earlier epidemiology studies by including a rigorous exposure assessment component, a nested case-control study of lung cancer and use of external and internal cohort rate comparisons. The primary research objectives include:

To investigate the total and cause-specific mortality experience of current and former workers as compared with corresponding national and regional populations and internallyderived control groups, with adjustment for potential confounding factors and focus on lung cancer.

To characterise the past and current working environment of subjects relative to work area, job title/function and potential for exposure to WCCo, as well as the component exposures: tungsten, tungsten carbide (without cobalt), carbon black, and cobalt;

To determine the relationship between level and duration of exposures and lung cancer mortality with adjustment for potential co-exposures, including information obtained on tobacco smoking habits via a nested case-control study.

Methods Our cohort comprises 10 manufacturing sites in the United States and nine sites in Europe, and represents three companies, five countries (US, Austria, Germany, Sweden and UK) and multiple manufacturing processes. The study will include separate and pooled analyses. The epidemiological and exposure assessment components of the study are coordinated by the University of Pittsburgh and the University of Illinois at Chicago, respectively. The study is funded by the International Tungsten Industry Association; the US record collection phase was supported in part by the Pennsylvania Department of Health.

Results We will report on current progress in the US component of the study and in our efforts to coordinate the international study. Progress in the European studies will be reported separately by the respective investigators.

\section{AN INTERNATIONAL HISTORICAL COHORT STUDY OF WORKERS IN THE HARD-METAL INDUSTRY - GERMAN COMPONENT}

